$\begin{array}{ll}\text { Research Square } & \begin{array}{l}\text { Preprints are preliminary reports that have not undergone peer review. } \\ \text { They should not be considered conclusive, used to inform clinical practice, } \\ \text { or referenced by the media as validated information. }\end{array}\end{array}$

\title{
Evaluation of rapid extraction methods coupled with Recombinase polymerase amplification assay for point-of-need diagnosis of Post-kala-azar-dermal leishmaniasis
}

\author{
Rajashree Chowdhury \\ International Centre for Diarrhoeal Disease Research Bangladesh \\ prakash ghosh ( $\sim$ dreamix888@gmail.com) \\ International Centre for Diarrhoeal Disease Research Bangladesh \\ Md. Anik Ashfaq Khan \\ International Centre for Diarrhoeal Disease Research Bangladesh \\ Faria Hossain \\ International Centre for Diarrhoeal Disease Research Bangladesh \\ Khaledul Faisal \\ International Centre for Diarrhoeal Disease Research Bangladesh \\ Rupen Nath \\ International Centre for Diarrhoeal Disease Research Bangladesh \\ James Baker \\ International Centre for Diarrhoeal Disease Research Bangladesh \\ Ahmed Abd El Wahed \\ Georg-August-Universitat Gottingen Universitatsmedizin \\ Shomik Maruf \\ International Centre for Diarrhoeal Disease Research Bangladesh \\ Proggananda Nath \\ Mymensingh Medical College \\ Debashis Ghosh \\ International Centre for Diarrhoeal Disease Research Bangladesh \\ Md. Masud-Ur Rashid \\ Heart Foundation \\ Malcolm S Duthie \\ Infectious Disease Research Institute \\ Dinesh Mondal \\ International Centre for Diarrhoeal Disease Research Bangladesh
}

Research article

Keywords: Post-kala-azar dermal leishmaniasis (PKDL), Point-of-need Diagnosis, DNA Extraction, Recombinase polymerase amplification (RPA), Real time PCR

Posted Date: November 11th, 2019

DOI: https://doi.org/10.21203/rs.2.17122/v1

License: @ (i) This work is licensed under a Creative Commons Attribution 4.0 International License. Read Full License 


\section{Abstract}

Introduction Post kala-azar dermal leishmaniasis (PKDL) usually develops as sequelae of visceral leishmaniasis (VL) and can manifest in multiple dermatological forms. Since PKDL patients harbor Leishmania donovani parasites and can potentially trigger inter-epidemic transmission of the disease, the success of kala-azar elimination programme could be jeopardized by these cases. Although several molecular methods with promising diagnostic efficacy have been developed to detect PKDL cases, albeit complicated and expensive DNA extraction methods limit their application in resource poor settings. To address this, in comparison to a reference DNA extraction method (Qiagen), we evaluated two rapid DNA extraction methods and determined their impact on the detection of the parasite DNA using our newly developed recombinase polymerase amplification (RPA) assay.

Methods Thirty suspected PKDL cases were enrolled after diagnosis by clinical examination and a positive rk39 strip test. DNA was extracted from three skin biopsy samples using either a spin column-based method (Qiagen) or one of two rapid DNA extraction methods, (Boil \& Spin (B\&S) and SpeedXtract (SE)). RPA and qPCR were subsequently performed with the extracted samples to detect L. donovani DNA.

Results Using DNA extracted by Qiagen method, the qPCR and RPA assays exhibited sensitivities of $86.7 \%$ and $93.3 \%$ respectively. In contrast, the sensitivity of RPA assay dropped to $76.7 \%$ and $63.3 \%$, respectively, when the B\&S and SE rapid extraction methods were performed. Despite this compromised sensitivity, B\&S-RPA technique yielded an excellent agreement with both Q-qPCR $(k=0.828)$ and Q-RPA $(k=0.831)$ techniques. Moreover, SE-RPA showed good agreement with Q-qPCR $(\mathrm{k}=0.755), \mathrm{Q}-\mathrm{RPA}(\mathrm{k}=0.692)$ and B\&S-RPA $(\mathrm{k}=0.635)$ assays. As expected, with all of the three DNA extraction methods, both qPCR and RPA assay showed absolute specificity.

Conclusions This study finding substantiates the superior diagnostic efficacy of Qiagen DNA extraction method over B\&S and SE method in detecting LD DNA through RPA assay from skin biopsy of PKDL patients. To apply these rapid DNA extraction methods in resource-constrained settings, further methodological refinement is warranted to improve DNA yield and purity through rigorous experiments.

\section{Background}

Post kala-azar dermal leishmaniasis (PKDL) is a sequelae of Leishmania donovani infection that mostly affects individuals after successful treatment for visceral leishmaniasis (VL)(1). PKDL usually manifests as macules (hypo-pigmented patches), papules and nodules or a combination of three known as polymorphic skin lesions mainly on the face, trunk, legs, arms and genital organ $(2,3)$. For unknown reasons the incidence of PKDL cases with different types of lesions varies across L. donovani endemic regions (2). In Sudan, 50-60\% of treated VL patients develop PKDL within 6 months, whereas, in Indian Subcontinent, PKDL is reported to develop in 5-10\% VL patients within 2-4 years after treatment(3-5). Surprisingly, the incidence rate of PKDL increases two fold within 5 years of completion of VL treatment (6). In addition, $15-20 \%$ of PKDL cases present without a documented history of VL, suggesting that these individuals may have had a prior sub-clinical L. donovani infection that was not detected (4). PKDL, unlike VL, is not life threatening if it remains untreated but PKDL patients often unfortunately experience stigma within their society $(7,8)$. Of further concern, the Leishmania parasites harboured within skin lesions of PKDL patients serve as the known reservoir of $V L$ and this plays a pivotal role in their inter-epidemic transmission through sandfly bites, particularly in the Indian subcontinent (9-11).

Kala-azar elimination programme (KEP) activities in the Indian subcontinent (ISC) have contributed to a remarkable decline in the incidence of kala-azar in recent years and the KEP is now considered to be in the consolidation phase. However, PKDL is identified as a potential threat to the sustained success of the programme and its ultimate goal of kala azar elimination. Proper diagnosis and management of PKDL has consequently been set as an essential component of the KEP (12,13). The control programme is facing challenges regarding early diagnosis and treatment of PKDL, however, because of its symptomatic resemblance to other skin diseases such as leprosy, vitiligo, secondary syphilis and sarcoidosis and the lack of sensitive filed-friendly diagnostic methods $(14,15)$. The lack of awareness and poor treatment seeking behavior of PKDL patients further complicate control activities $(16,17)$.

Currently diagnosis of PKDL relies on clinical assessments with support from parasitological approaches (2). Direct demonstration of Leishmania amastigotes in either slit skin or skin biopsy smear provides 60-100\% sensitivity in nodular lesions but has poor sensitivity in macular lesions (7-50\%) (1820). Furthermore, several antibody-based serological methods such as direct agglutination test (DAT), enzyme linked immunosorbent assay (ELISA) and rK39based rapid diagnostic tests (RDT) have been considered as ancillary diagnostic tests for PKDL diagnosis, because all of the treated VL patients give positive result for antibody based methods, even after being cured $(14,21)$. In contrast, molecular methods can detect $L$. donovani DNA, and several conventional as well as real time PCR assays have been developed with highly sensitivities and specificities for laboratory diagnosis of both VL and PKDL (18,22). These methods can help confirm the diagnosis of PKDL in $40-94 \%$ of clinically suspected individuals $(4,23)$, and we previously developed a promising real time PCR assay for the diagnosis of PKDL that provided excellent sensitivity (91.2\%) for macular PKDL cases in endemic regions of Bangladesh (18). The application of qPCR in resource limited settings, including primary and secondary health-care facilities, is however challenging because it requires a well-equipped laboratory, trained personnel and reliable storage conditions of the reagents. Therefore, the need for a user-friendly, design-locked and field-feasible diagnostic method for PKDL detection remains. On this regard, the recombinase polymerase amplification (RPA) assay has recently emerged as a novel alternative isothermal amplification technology for the detection of nucleic acid $(24,25)$ with the potential to overcome the limitations of poor resource settings. RPA provides results faster than conventional and even real time-PCR, despite amplifying nucleic acid at a constant temperature (42 $\mathrm{C}$ ), and requiring less expensive and simpler equipment (24). Due to the many advantages of the RPA assay, we developed a RPA assay for detection of Leishmania donovani (LD) parasites that showed absolute sensitivity and specificity in correspondence with real-time PCR (26).

In addition to the detection method, an important parameter for the optimum sensitivity of molecular diagnostic approaches is the extraction of high-quality genomic DNA extraction from clinical specimens. Generally, Spin column-based extraction method produces pure DNA but requires use of high-speed centrifugation with enhanced washing steps, which is costly and usually not feasible in field settings. On the other hand, the SpeedXtract (SE) method that has been incorporated with RPA assay in several studies involves a magnetic bead-based lysis protocol to avoid the creation of aerosols and the use of a high- 
speed centrifuge. The SE method has not, however, been evaluated using skin samples. Alternatively, we found a relatively simple DNA extraction method based on an in-house lysis buffer to be suitable for Loop-mediated isothermal assays $(27,28)$. In an effort to develop a field-friendly diagnostic algorithm for detecting $L$. donovani DNA in skin samples from PKDL patients we therefore assessed various nucleic acid extraction techniques in combination with an RPA assay.

\section{Methods And Materials Study sites and participants:}

The study entailed both field and laboratory activities. Field activities were performed at Surja Kanta Kala-azar Research Centre (SKKRC), Mymensingh, Bangladesh, a region highly endemic for VL, and Laboratory activities at Emerging Infections and Parasitology, icddr,b, Dhaka, following the approval of the International Centre for Diarrhoeal Disease Research, Bangladesh (icddr,b) Institutional Review Board (IRB) (PR-17041). In total thirty treatment seeking, suspected PKDL cases residing in the endemic zone were enrolled at Surya kanta kala-azar research centre (SKKRC), the only specialized hospital for treatment of VL, PKDL, and their associated complications. The majority of the recruited PKDL patient had a history of VL and all exhibited characteristic skin rashes. All PKDL patients were positive in rk39 RDT and were diagnosed based on clinical characteristics by the hospital physician. Following initial examination, each patient was invited to participate in the study and written informed consent was obtained from either the participant or the legal guardian of children participants before samples were collected. Following standard procedures, the study physician collected three $3 \mathrm{~mm}$ skin biopsy samples from each participant. Each biopsy was preserved in NET buffer for subsequent DNA extraction. All PKDL patients were referred for treatment following national guidelines and each was found to be responsive to treatment. To determine the specificity of our investigative assays thirty DNA samples were extracted from buffy coat of cured VL patients and were also subjected to laboratory analyses.

\section{DNA extraction from clinical specimen:}

\section{DNA was isolated following three DNA extraction methods:}

Spin column-based method: DNA was extracted using a QIAamp DNA tissue \& blood mini kit (Qiagen, Hilden, Germany) according to the manufacturer's protocol with a minor modification: skin biopsy materials were kept at $37^{\circ} \mathrm{C}$ overnight after addition of ATL buffer and protease $\mathrm{K}$. The following day, the material was homogenized then incubated at $56^{\circ} \mathrm{C}$ for two hours before purification.

SpeedXtract Extraction (SE) method: A simple and rapid blood lysis protocol (SpeedXtract, Qiagen, Hilden, Germany) was modified to suit DNA extraction from skin as follows: $100 \mu$ l of Buffer SL and $30 \mu$ of Suspension A (SpeedXtract, Qiagen, Lake Constance, Germany) was added with 3 mm skin punch biopsy in a $2 \mathrm{ml}$ tube and was mixed thoroughly by vortexing for 10 seconds. Thereafter, the mix was incubated at $95^{\circ} \mathrm{C}$ for 10 minutes and after incubation the skin biopsy was pressed with grinding pestle and mixed by vortexing. The mix was incubated at $95^{\circ} \mathrm{C}$ for another 10 minutes then the tube was transferred to a magnetic stand and incubated at room temperature for 1 minute. Finally, the supernatant was carefully transferred to a new tube.

Boil \& Spin (B \& S) method: Skin biopsy materials were kept in $37^{\circ} \mathrm{C}$ for overnight after addition of an in-house prepared simple lysis buffer (400 mM NaCl, 40 $\mathrm{mM}$ Tris pH 6.5, 0.4\% SDS) (28) following addition of protease K (Qiagen, Hilden, Germany). The following day, the skin materials were homogenized then incubated at $70^{\circ} \mathrm{C}$ for 15 minutes. After incubation, the mixture was vortexed, spun and incubated for 5 minutes at $95^{\circ} \mathrm{C}$ before centrifugation for 3 min at $10,000 \mathrm{~g}$. After centrifugation, $30 \mu \mathrm{L}$ of clear supernatant was transferred to the Dilution Tube containing $345 \mu \mathrm{L}$ of PCR grade water.

\section{DNA purity and concentration:}

To assess the purity of each extracted DNA sample, OD values at $260 \mathrm{~nm}$ and $280 \mathrm{~nm}$ was measured by a Thermo Scientific Nanodrop ${ }^{\text {TM }} 2000$ Spectrophotometer (Thermo Scientific, Germany) and the ratio calculated (the standard ratio for purified DNA ranges between 1.8-2.0). Subsequently, DNA concentration/quantity was determined from the OD value at $260 \mathrm{~nm}$ following the standard method (29).

\section{Molecular detection of LD-DNA:}

Recombinase polymerase amplification (RPA) assay: The RPA assay was performed with the extracted DNA samples following the previously published

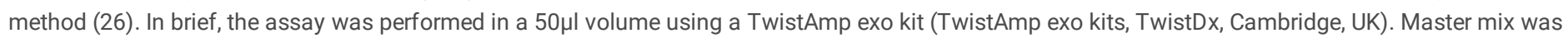
prepared in a tube with $420 \mathrm{nM}$ of RPA primer, $120 \mathrm{nM}$ of RPA Probe, $1 \mathrm{x}$ rehydration buffer and was added to the RPA lyophilized pellet. Then, 14 mM Mg acetate was pipetted into the tube lids. Subsequently, template DNA was added to the tubes and the tube was closed and mixed well. The tubes were immediately placed into the tubescanner (Twista, TwistDx, Cambridge, UK) and incubated for $15 \mathrm{~min}$ at $42{ }^{\circ} \mathrm{C}$. The emitted fluorescence signals were measured at 20 s intervals. A combined threshold and first derivative analysis were used for signal interpretation. The total reaction time for RPA was approximately 20 minutes.

Real time PCR: The real time PCR was also performed by a previously published method (30). Briefly, Taqman primers and probes were designed targeting conserved region of Leishmania REPL repeats (L42486.1) specific for L. donovani and L. infantum and synthesized by Applied Biosystems(30). Briefly, a 20 $\mu \mathrm{L}$ reaction mix was prepared containing $5 \mu \mathrm{L}$ template, $10 \mu \mathrm{L}$ of TaqMan ${ }^{\circledR}$ Gene Expression Master Mix $(2 \mathrm{X}), 1 \mu \mathrm{L}$ pre-ordered primer-probe mix and PCR 
grade water. Amplification was performed on a Bio-rad CFX96 icycler system with following reaction conditions: 10 min at $95^{\circ} \mathrm{C}$, followed by 45 cycles of 15 seconds at $95^{\circ} \mathrm{C}$ and $1 \mathrm{~min}$ at $60^{\circ} \mathrm{C}$. Samples with cycle threshold $(\mathrm{Ct})>40$ were considered negative. The total reaction time for real time PCR was approximately 120 minutes.

\section{Reagent Cost and time analysis:}

The reagent costs associated with this study were assessed similarly to previous studies (31-33). We estimated the cost of each qPCR or RPA reaction including DNA extraction for individual sample, where only the operational costs including supplies, kit and reagent cost were under consideration. Costs for infrastructure, labor, training and supervision were not included in the calculation. The time required for each assay was estimated through inclusion of sample processing time prior to each respective DNA extraction method and detection time associated with either qPCR or RPA.

\section{Statistical analysis:}

Parametric and non-parametric tests were performed based on the distribution of data. Kappa and McNemar's test were performed to determine the concordance and discordance among three extraction methods in combination with RPA assay. Standard statistical formulas were followed to determine the sensitivity and specificity of the test with $95 \% \mathrm{Cl}$. Furthermore, Receiver operating characteristic (ROC) curve analysis was performed to determine the accuracy of each of the extraction method when coupled with RPA/qPCR assay. All statistical analyses were performed using SPSS (Version 20.0) and GraphPad Prism (Version 8.1.2). P value $<0.05$ was considered as statistically significant.

\section{Results}

\section{Participants' indices:}

Among the 30 clinically confirmed PKDL cases recruited, $60 \%$ were male and the mean age of the participants was $26.47 \pm 12.41$ years. Previous history of VL was reported in $93.3 \%$ of the PKDL patients, and $23.3 \%$ were relapse cases (Table-1). The clinical examination confirmed 22 (73.3\%) as macular cases whereas the remaining cases presented with either nodular $(6.7 \%)$ or mixed $(20 \%)$ lesions.

\section{Extraction method-based performance of RPA/qPCR assay:}

The qPCR assay detected 26 out of 30 clinically confirmed PKDL patients with a sensitivity of $86.67 \%$, when DNA was extracted with the Spin column based (Qiagen) method. Likewise, the RPA assay showed elevated performance with a sensitivity of $93.33 \%$ when same DNA extraction method was followed. On the other hand, when the B\&S (23/30) and SE (19/30) methods were performed, the RPA assay showed compromised diagnostic efficacy with a sensitivity of $76.7 \%$ and $63.3 \%$ respectively (Table-2). As expected, Q-RPA (20/22) and Q-qPCR (18/22) exhibited a considerably higher positive rate (90.9\% and 81.8\%) than B\&S-RPA and SE-RPA (69.6\% and 59.1\%) among macular PKDL cases. In addition, all nodular and mixed cases were detected by both Q-RPA and Q-qPCR, whereas one nodular case was not detected by B\&S-RPA and two mixed cases remained undetected by SE-RPA assay. Both qPCR and RPA assays showed absolute specificity for all of the extraction methods.

The ROC analysis showed superior diagnostic accuracy of Q-RPA as presented by the highest value of area under the curve (AUC) (AUC $=0.967)$. As the least efficacious technique, SE-RPA gave the lowest AUC value (AUC = 0.817) (Fig 2C). Notwithstanding the poor performance, B\&S-RPA presented an excellent agreement with both Q-qPCR $(\mathrm{k}=0.828)$ and Q-RPA $(\mathrm{k}=0.831)$ technique whereas SE-RPA showed good agreement with Q-qPCR $(\mathrm{k}=0.755)$, Q-RPA $(\mathrm{k}=$ $0.692)$ and B\&S-RPA $(k=0.635)$ assay $($ Table-3) techniques. Furthermore, SE-RPA showed significant discordance with both $Q-q P C R(p=0.02)$ and $Q-R P A(p$ $=0.004)($ Table-3). Overall, among 30 clinically diagnosed PKDL cases, 16 cases were found to be pan-positive and 2 cases were pan-negative.

\section{Efficiency of DNA extraction methods:}

The DNA concentration was highest for the SpeedXtract method with an average concentration of $140.7 \pm 50.5 \mathrm{ng} / \mu \mathrm{l}$ compared to the Qiagen ( $21.6 \pm 10.4 \mathrm{ng} /$ $\mu \mathrm{l})$ and Boil \& Spin $(22.9 \pm 9.3 \mathrm{ng} / \mu \mathrm{l})$ method (Table-2). However, the Spin column-based extraction method (Qiagen) gave most purified DNA with a mean OD $260 / 280$ ratio of $1.85 \pm 0.09$ while the SE extraction method gave least purified DNA with a mean OD $260 / 280$ ratio of $0.77 \pm 0.22$ (Table-2). To be noted, a significant difference was found between RPA positive and negative samples regarding the DNA concentration $(p<0.001)$ and purity $(p<0.0001)$ for $B \& S$ method which was not observed for two other methods (Figure 2A and 2B).

\section{Comparison of assay time and cost-effectiveness:}

The cost and time estimation analysis showed that the cost and time required for the Spin column based (Qiagen) DNA extraction method coupled with qPCR assay were approximately $\$ 16.5$ and $17 \mathrm{hrs}$ respectively for each sample, whereas RPA assay has a lower cost per sample of $\$ 7.5$ and requires less performance time (15hrs 20 mins). We found the B\&S-RPA assay as the least expensive (app. \$5) assay, whereas SE-RPA assay was less time-consuming ( 40 min) compared to Q-RPA (15 hrs 20 mins) and B\&S-RPA (13 hrs) assay. 


\section{Discussion}

Tremendous declines in the incidence/rate of $\mathrm{VL}$ and its mortality due to the initiatives of KEP indicate substantial advancement towards the elimination of kala-azar from Indian sub-continent (ISC), including Bangladesh. The regional VL initiative is actively pursuing the elimination targets by 2020 and Bangladesh has already succeeded in reaching the targets in over $90 \%$ of its endemic sub-districts (34-36). Following the success of the attack phase, the KEP has moved to the consolidation phase with the aim of identifying and controlling potential sources of $L$. donovani infection in endemic areas. Our recent studies have demonstrated that, in addition to VL patients PKDL cases can competently transmit the parasites to generate new VL cases (10). PKDL cases are now considered as important reservoirs for parasites and are deemed to be key contributors to interepidemic disease transmission. Therefore, early diagnosis and treatment of PKDL should be prioritized as additional means to control the transmission of Leishmania parasites and to ensure elimination is sustained. In a recent review, Zijlstra et al suggested that validation and implementation of diagnostic methods, including qPCR or isothermal amplification technique, are essential for diagnosis of PKDL to sustain the success of VL elimination efforts in the ISC (11). In addition to clinical parameters, qPCR is considered to be the most promising method for diagnosis and assessments of cure $(18,22)$. RPA assay has, however, shown comparable diagnostic efficacy to qPCR while surpassing PCR-based molecular methods with multitude of practical and technological advantages (26). A simple and inexpensive method of DNA extraction would make RPA assay even more feasible and sustainable in poor resource settings. We therefore evaluated three DNA extraction methods together with RPA assay and compared to the reference DNA extraction method (Qiagen) coupled with the qPCR assay to determine if we could generate a field amenable and cost-effective diagnostic method for diagnosis of PKDL.

Our data indicate that, relative to other extraction methods, considerably higher detection rates are achieved for RPA (93.3\%) and qPCR (86.7\%) assay when DNA extraction was performed with the Spin column-based method. We found similar sensitivity ( $83.4 \%-96.1 \%)$ for qPCR in our previous study where only macular cases with low parasite burden were included (18). In the current study all nodular and mixed PKDL cases were positive in both RPA and qPCR, a result that is consistent with their higher parasite abundance (13). These data clearly indicate the promising diagnostic efficacy of Q-RPA assay in detecting Leishmania parasites in skin biopsies from PKDL patients. However, the sensitivity of RPA assay was compromised, when the rapid DNA extraction methods such as Boil \& Spin (B\&S) and SpeedXtract (SE) were used. Although several studies have reported excellent performance of isothermal amplification based assays such as LAMP and RPA assay conducted with on nucleic acid extracted from whole blood sample by Boil \& Spin and SpeedXtract methods (26-28), our finding is similar to that of an earlier study performed in Sri Lanka on CL patients that reported 65.5\% sensitivity of SE-RPA (37).

The comparatively lower sensitivity of Boil \& Spin and SpeedXtract DNA extraction methods in RPA assay might be attributed to the inherent limitations of these techniques. Notably, the absence of washing steps and deproteination agent such as proteinase K (SpeedXtract) leads to poor quality DNA with impurities that may inhibit the activity of recombinase and polymerase enzymes in RPA assay (38). In addition, the 2.5 times lower A260/A280 ratio in SE method provides another empirical demonstration of the underperformance of RPA assay due to the protein impurities in the DNA. Moreover, we noted that skin samples were only partially digested at the end of recommended ten minutes in SE method (notably, the digestion time for two other methods was longer). Surprisingly, we found a significant difference in A260/A280 ratio ( $<<0.0001)$ of DNA being extracted by Boil \& Spin method between RPA positive and negative samples, suggesting promise for this extraction method by increasing the DNA yield. Considering the cost and time for each of the assays, we estimated that the B\&S-RPA assay is 3-fold inexpensive (at \$5 per sample) compared to Q-qPCR (at \$16.5 per sample). On the other hand, SE-RPA assay was far less time-consuming and can produce results significantly faster (within $40 \mathrm{~min}$ ) than other combinations. Last but not the least, B\&S-RPA assay showed excellent agreement with Q-RPA and Q-qPCR assays, and SE-RPA assay presented good agreement with Q-qPCR, Q-RPA and B\&S-RPA assays (table$3)$, further indicating the promise of B\&S-RPA and SE-RPA assays in detecting LD parasite from clinical samples.

The major limitation of this study is that we performed RPA and QPCR assay with DNA extracted by three different DNA Extraction methods using different skin biopsies. This might have generated some variance, and an over-or underestimation of the performance of any of the methods, as the parasites are not evenly distributed in the lesions of PKDL patients (5). The skin biopsy procedure is invasive and requires surgical set-up and this limits the ability to collect multiple biopsies without negatively impacting patient participation and use as an active field-based case detection method. Several recent studies have indicated the satisfactory diagnostic efficacies of the less invasive slit skin, micro biopsy, and fine needle biopsy methods $(20,39,40)$. Further evidence is required, however, before these invasive sample collection procedures can be applied for diagnosis of PKDL. Another limitation to our study is that, due to ethical issues, we used buffy coat samples from cured VL patients instead of the skin biopsy samples to determine assay specificity. Our previous study provided a basis for the use of blood sample for evaluating specificity of an index method in PKDL diagnostic studies (18).

Considering the diagnostic performance, operational cost and feasibility, our data indicate that a Spin column based (Qiagen) DNA extraction method coupled with RPA assay can be routinely performed as an alternative of qPCR for diagnosis of PKDL cases. In addition, we recommend a refined B\&S method as an alternate method to the reference DNA extraction method. Further modification of the SpeedXtract method to achieve better sensitivity could generate a SERPA assay that could be used as a point-of-contact tool for rapid diagnosis of PKDL. Further large-scale studies are both warranted and required to generate a Q-RPA assay that can be used for the molecular diagnosis of leishmaniasis involving dermatological complications.

\section{Conclusions}

The findings of this study represent the superior diagnostic performance of Qiagen DNA extraction method over Boil\&Spin (B\&S) and SpeedXtract (SE) method in detecting LD DNA through RPA assay from skin biopsy of PKDL patients. We recommend, a Spin column based (Qiagen) DNA extraction method coupled with RPA assay, as a surrogate mode of diagnosis of PKDL which can be routinely performed as an alternative of qPCR. We believe our findings, and the recommendations we make from them, could help policy makers adopt a cost- effective diagnostic method for PKDL that could be implemented in resource-limited settings to help the KEP sustain their successes.

\section{Abbreviations}


AUC: Area under the curve

Cl: Confidence interval

B\&S: Boil \& Spin

DAT: Direct agglutination test

DNA: Deoxyribonucleic acid

ELISA: Enzyme linked immunosorbent assay

ISC: Indian subcontinent

KEP: Kala-azar elimination programme

PKDL: Post-kala-azar dermal leishmaniasis

qPCR: Real-time or quantitative polymerase chain reaction

RPA: Recombinase polymerase amplification

ROC: Receiver operating curve

SE: SpeedXtract

VL: Visceral leishmaniasis

Declarations

\section{Ethics approval and consent to participate}

This study was approved by the International Centre for Diarrhoeal Disease Research, Bangladesh (icddr,b) Institutional Review Board (IRB), research protocol number PR-17041. Informed written consent was collected from each participant or the legal guardian in the case of children.

\section{Consent for Publication}

Not applicable

\section{Availability of data and materials}

The datasets used and/or analysed during the current study are available from the corresponding author on reasonable request.

\section{Competing interests}

The authors declare that they have no competing interests.

\section{Funding:}


This study was awarded by the Swedish International Development Cooperation Agency (SIDA) and the award no is GR-01455.The authors alone are responsible for the views expressed in this manuscript and the funding sources had no role in the study design, collection, analysis and interpretation of the data, preparation of the manuscript, or the decision to submit for publication.

\section{Authors' contributions}

PG, DM, PN, MSD and AAEW conceived and designed the study. PG, RC, FH, MAAK, KF, SM, DG, JB, MR and RN performed the experiments and maintained the data source. PG, RC and MAAK performed the statistical analysis. RC and PG drafted the manuscript. PG, DM, MSD and AAEW revised the manuscript. All authors read and approved the final version of the manuscript.

\section{Acknowledgements:}

We are grateful to all of the participants for their valuable participation in this study. The authors are grateful to core donors which provide unrestricted support to icddr,b. Current donors include the Government of the People's Republic of Bangladesh; Global Affairs Canada GAC), Canada; Swedish International Development Cooperation Agency (Sida); and the Department for International Development (UKAid). Prakash Ghosh was supported as a Clinical Research and Development Fellow (CRDF) by UNICEF/UNDP/World Bank/WHO Special Programme for Research and Training in Tropical Diseases (TDR) during the study period.

\section{References}

1. Verma S, Bhandari V, Avishek K, Ramesh V, Salotra P. Reliable diagnosis of post-kala-azar dermal leishmaniasis ( PKDL) using slit aspirate specimen to avoid invasive sampling procedures. 2013;18(3):268-75.

2. Mondal D, Khan MGM. Recent advances in post-kala-azar dermal leishmaniasis. Curr Opin Infect Dis [Internet]. 2011;24(5):418-22. Available from: http://www.ncbi.nlm.nih.gov/pubmed/21885919

3. Zijlstra EE, Musa AM, Khalil EAG, El Hassan IM, El-Hassan AM. Post-kala-azar dermal leishmaniasis. Lancet Infect Dis. 2003;3(2):87-98.

4. Rahman KM, Islam S, Rahman MW, Kenah E, Galive CM, Zahid MM, et al. Increasing Incidence of Post-Kala-Azar Dermal Leishmaniasis in a PopulationBased Study in Bangladesh. Clin Infect Dis [Internet]. 2010 Jan 1;50(1):73-6. Available from: http://dx.doi.org/10.1086/648727

5. Ganguly S, Saha P, Chatterjee M, Roy S. PKDL-A Silent Parasite Pool for Transmission of Leishmaniasis in Kala-azar Endemic Areas of Malda District, West. 2015;1-13.

6. Islam S, Kenah E, Ashraful M, Bhuiyan A, Rahman KM, Goodhew B, et al. Clinical and Immunological Aspects of Post-Kala-Azar Dermal Leishmaniasis in Bangladesh. 2013;89(2):345-53.

7. Mondal D, Hasnain MG, Hossain MS, Ghosh D, Ghosh P, Hossain H, et al. Study on the safety and efficacy of miltefosine for the treatment of children and adolescents with post-kala-azar dermal leishmaniasis in Bangladesh, and an association of serum vitamin $\mathrm{E}$ and exposure to arsenic with post-kala-azar dermal leishmaniasis: A. BMJ Open. 2016;6(5):1-9.

8. Garapati P, Pal B, Siddiqui NA, Bimal S, Das P, Murti K, et al. Knowledge, stigma, health seeking behaviour and its determinants among patients with post kalaazar dermal leishmaniasis, Bihar, India. PLoS One [Internet]. 2018;13(9):e0203407. Available from:

http://dx.plos.org/10.1371/journal.pone.0203407\%0Ahttp://www.ncbi.nlm.nih.gov/pubmed/30192805\%0Ahttp://www.pubmedcentral.nih.gov/articlerend artid = PMC6128567

9. Molina R, Ghosh D, Carrillo E, Monnerat S, Bern C, Mondal Di, et al. Infectivity of Post-Kala-azar Dermal Leishmaniasis Patients to Sand Flies: Revisiting a Proof of Concept in the Context of the Kala-azar Elimination Program in the Indian Subcontinent. Clin Infect Dis. 2017;65(1):150-3.

10. Mondal D, Bern C, Ghosh D, Rashid M, Molina R, Chowdhury R, et al. Quantifying the infectiousness of post-kala-azar dermal leishmaniasis towards sandflies. Clin Infect Dis. 2018;

11. Zijlstra EE, Alves F, Rijal S, Arana B, Alvar J. Post-kala-azar dermal leishmaniasis in the Indian subcontinent: A threat to the South-East Asia Region Kalaazar Elimination Programme. PLoS Negl Trop Dis. 2017;11(11):e0005877.

12. WHO. Regional Strategic Framework for Elimination of Kala-azar from the South-East Asia. Sea-Cd-239 [Internet]. 2014;24. Available from: http://apps.searo.who.int/pds_docs/B4870.pdf?ua = 1\%0Ahttp://www.searo.who.int/entity/world_health_day/2014/KA_CD239.pdf

13. Moulik S, Chaudhuri SJ, Sardar B, Ghosh M, Saha B, Das NK, et al. Monitoring of Parasite Kinetics in Indian Post-Kala-azar Dermal Leishmaniasis. Clin Infect Dis. 2017;66(3):404-10.

14. Salotra P, Kaushal H, Ramesh V. Kala Azar in South Asia. 2016;(VI):7-22. Available from: http://link.springer.com/10.1007/978-3-319-47101-3

15. Adams ER, Versteeg I, Leeflang MMG. Systematic Review into Diagnostics for Post-Kala-Azar Dermal Leishmaniasis (PKDL). $2013 ; 2013$.

16. Huda MM, Hirve S, Siddiqui NA, Malaviya P, Banjara MR, Das $P$, et al. Active case detection in national visceral leishmaniasis elimination programs in Bangladesh, India, and Nepal: feasibility, performance and costs. BMC Public Health. 2012;12(1):1001.

17. Basher A, Nath P, Nabi SG, Selim S, Rahman F, Sutradhar SR, et al. A Study on Health Seeking Behaviors of Patients of Post-Kala-Azar Dermal Leishmaniasis. 2015;2015.

18. Ghosh P, Hasnain G, Hossain F, Khan AA, Chowdhury R, Faisal K, et al. Evaluation of Real-time PCR for Diagnosis of Post-Kala-azar Dermal Leishmaniasis in Endemic Foci of Bangladesh. 2018;4-9. 
19. Salotra P, Singh R. Challenges in the diagnosis of post kala-azar dermal leishmaniasis. Indian J Med Res. 2006;123(3):295.

20. Verma N, Singh D, Pandey K, Nand V, Das R, Lal CS, et al. Comparative Evaluation of PCR and Imprint Smear Microscopy Analyses of Skin Biopsy Specimens in Diagnosis of Macular, Papular, and Mixed Papulo-Nodular Lesions of Post-Kala-Azar Dermal. 2013;51(12):4217-9.

21. Rijal S, Soto A, Menten J, Boelaert M. A meta-analysis of the diagnostic performance of the direct agglutination test and rK39 dipstick for visceral leishmaniasis. 2006;(August).

22. Hossain F, Ghosh P, Khan AA, Duthie MS, Vallur C, Picone A, et al. Real-time PCR in detection and quantitation of Leishmania donovani for the diagnosis of Visceral Leishmaniasis patients and the monitoring of their response to treatment. 2017;1-16.

23. Srivastava P, Mehrotra S, Tiwary P, Chakravarty J, Sundar S. Diagnosis of Indian visceral leishmaniasis by nucleic acid detection using PCR. PLoS One. 2011;6(4):e19304.

24. Faye O, Faye O, Soropogui B, Patel P, El WAA, Loucoubar C, et al. Development and deployment of a rapid recombinase polymerase amplification Ebola virus detection assay in Guinea in 2015. Eurosurveillance. 2015;20(44).

25. Piepenburg O, Williams CH, Stemple DL, Armes NA. DNA detection using recombination proteins. PLoS Biol. 2006;4(7):e204.

26. Mondal D, Ghosh P, Khan MAA, Hossain F, Böhlken-Fascher S, Matlashewski G, et al. Mobile suitcase laboratory for rapid detection of Leishmania donovani using recombinase polymerase amplification assay. Parasit Vectors. 2016;9(1).

27. Mukhtar M, Ali SS, Boshara SA, Albertini A, Bessell P, Mori Y, et al. Sensitive and less invasive confirmatory diagnosis of visceral leishmaniasis in Sudan using loop-mediated isothermal amplification. 2018;1-14.

28. Hopkins H, González IJ, Polley SD, Angutoko P, Ategeka J, Asiimwe C, et al. Highly Sensitive Detection of Malaria Parasitemia in a Malaria-Endemic Setting: Performance of a New Loop-Mediated Isothermal Ampli fi cation Kit in a Remote Clinic in Uganda. 2013;208:645-52.

29. Desjardins P, Conklin D. NanoDrop microvolume quantitation of nucleic acids. JoVE (Journal Vis Exp. 2010;(45):e2565.

30. Vallur AC, Duthie MS, Reinhart C, Tutterrow Y, Hamano S, Bhaskar KRH, et al. Biomarkers for intracellular pathogens: establishing tools as vaccine and therapeutic endpoints for visceral leishmaniasis. Eur Soc Clin Infect Dis [Internet]. 2015;20(6):0374-83. Available from: http://dx.doi.org/10.1111/14690691.12421

31. Burke RM, McKenna JP, Cox C, Coyle P V, Shields MD, Fairley DJ. A comparison of different pre-lysis methods and extraction kits for recovery of Streptococcus agalacticae (Lancefield group B Streptococcus) DNA from whole blood. J Microbiol Methods. 2016;129:103-8.

32. de Assis TSM, Azeredo-da-Silva ALF, Werneck GL, Rabello A. Cost-effectiveness analysis of diagnostic tests for human visceral leishmaniasis in Brazil. Trans R Soc Trop Med Hyg. 2016;110(8):464-71.

33. Xing W, Yu X, Feng J, Sun K, Fu W, Wang Y, et al. Field evaluation of a recombinase polymerase amplification assay for the diagnosis of Schistosoma japonicum infection in Hunan province of China. BMC Infect Dis. 2017;17(1):164.

34. Sundar S, Singh OP, Chakravarty J. Visceral leishmaniasis elimination targets in India, strategies for preventing resurgence. Expert Rev Anti Infect Ther. 2018;16(11):805-12.

35. Singh OP, Hasker E, Boelaert M, Sundar S. Elimination of visceral leishmaniasis on the Indian subcontinent. Lancet Infect Dis. 2016;16(12):e304-9.

36. Hirve S, Kroeger A, Matlashewski G, Mondal D, Banjara R, Das P, et al. Towards elimination of visceral leishmaniasis in the Indian subcontinentTranslating research to practice to public health. 2017;1-25.

37. Gunaratna G, Manamperi A, Böhlken-fascher S, Wickremasinge R, Gunawardena K, Yapa B, et al. Evaluation of rapid extraction and isothermal amplification techniques for the detection of Leishmania donovani DNA from skin lesions of suspected cases at the point of need in Sri Lanka. $2018 ; 1-7$.

38. Daher RK, Stewart G, Boissinot M, Bergeron MG. Review Recombinase Polymerase Amplification for Diagnostic Applications. 2016 ;958.

39. David O, Abbasi I, Zion B, Skrip L, Hailu A, Jaffe C, et al. Minimally invasive microbiopsies: a novel sampling method for identifying asymptomatic, potentially infectious carriers of Leishmania donovani. Int J Parasitol [Internet]. 2017;47(10-11):609-16. Available from: http://dx.doi.org/10.1016/j.ijpara.2017.02.005

40. North F. Fine-Needle Sampling Provides Appreciable Diagnostic Yield in Lesions of Post Kala Azar Dermal Leishmaniasis: Analysis of Four Cases. 2014; (February 2013):525-9.

\section{Tables}

Table 1: Clinical and Demographic parameters of the PKDL patients enrolled in the study ( $\mathrm{N}=30$ ).

\begin{tabular}{|c|c|c|}
\hline \multicolumn{2}{|l|}{ Variable } & Value \\
\hline \multicolumn{2}{|l|}{ Male, n (\%) } & $18 / 30(60 \%)$ \\
\hline \multicolumn{2}{|c|}{ Age in years, mean $\pm S D$} & $26.47 \pm 12.41$ \\
\hline \multicolumn{2}{|c|}{ Children (<17years), n (\%) } & $9 / 30(30 \%)$ \\
\hline \multicolumn{2}{|c|}{ Adults (>17 years), $\mathrm{n}(\%)$} & $21 / 30(70 \%)$ \\
\hline \multicolumn{2}{|c|}{ Past History of VL, $\mathrm{n}(\%)$} & $28 / 30(93.3 \%)$ \\
\hline \multicolumn{2}{|c|}{ Past History of PKDL, n (\%) } & $7 / 30(23.3 \%)$ \\
\hline \multirow{3}{*}{ Type of Rash, $n$ (\%) } & Macular & $22 / 30(73.3 \%)$ \\
\hline & Nodular & $2 / 30(6.7 \%)$ \\
\hline & Mixed & $6 / 30(20 \%)$ \\
\hline
\end{tabular}


Table 2: Analysis of performance for three DNA extraction methods with qPCR and RPA assay

\begin{tabular}{|c|c|c|c|c|c|c|c|c|c|}
\hline \multirow[t]{2}{*}{$\begin{array}{l}\text { DNA Extraction } \\
\text { Methods }\end{array}$} & \multirow{2}{*}{$\begin{array}{c}\text { Mean DNA conc. } \\
\text { (ng/pl) } \\
{[95 \% \mathrm{CI}]} \\
\mathrm{N}=30\end{array}$} & \multirow{2}{*}{$\begin{array}{c}\text { Mean OD } 260 / 280 \\
\text { ratio } \\
{[95 \% \mathrm{CI}]} \\
\mathrm{N}=30\end{array}$} & \multirow{2}{*}{$\begin{array}{l}\text { Sensitivity } \\
\text { of qPCR } \\
\text { [95\% CI] } \\
\text { (Pos/Neg) }\end{array}$} & \multirow{2}{*}{$\begin{array}{c}\text { Sensitivity of } \\
\text { RPA } \\
\text { [95\% CI] } \\
\text { (Pos/Neg) }\end{array}$} & \multirow{2}{*}{$\begin{array}{c}\text { Specificity of Q-qPCR } \\
\text { and RPA } \\
{[95 \% \mathrm{CI}]} \\
\text { (Pos/Neg) }\end{array}$} & \multicolumn{2}{|c|}{$\begin{array}{c}\text { App. Time } \\
\text { (per sample) }\end{array}$} & \multicolumn{2}{|c|}{$\begin{array}{l}\text { App. Cost } \\
\text { (in \$ per } \\
\text { sample) }\end{array}$} \\
\hline & & & & & & qPCR & RPA & qPCR & RPA \\
\hline $\begin{array}{l}\text { Spin-column } \\
\text { method } \\
\text { (Qiagen) }\end{array}$ & $\begin{array}{c}21.6 \pm 10.4 \\
{[17.71-25.49]}\end{array}$ & $\begin{array}{c}1.85 \pm 0.09 \\
{[1.81-1.88]}\end{array}$ & $\begin{array}{c}86.67 \% \\
{[69.28 \%-96.24 \%]} \\
(26 / 4)\end{array}$ & $\begin{array}{c}93.33 \% \\
{[77.93 \%-99.18 \%]} \\
(28 / 2)\end{array}$ & & $17 \mathrm{hrs}^{\mathrm{a}}$ & $\begin{array}{c}15 \text { hrs } 20 \\
\min ^{\mathrm{c}}\end{array}$ & $16.5^{\mathrm{b}}$ & $\begin{array}{l}7.5 \\
\mathrm{c}, \mathrm{e}\end{array}$ \\
\hline $\begin{array}{l}\text { Boil \&Spin } \\
\text { (B\&S) }\end{array}$ & $\begin{array}{c}22.9 \pm 9.3 \\
{[19.47-26.44]}\end{array}$ & $\begin{array}{c}0.91 \pm 0.11 \\
{[0.87-0.95]}\end{array}$ & N/A & $\begin{array}{c}76.67 \% \\
{[57.72 \%-90.07 \%]} \\
(23 / 7)\end{array}$ & & $15 \mathrm{hrs}^{\mathrm{a}, \mathrm{b}}$ & 13 hrs $^{b}$ & $14.0^{\mathrm{d}}$ & $5.0^{\mathrm{d}}$ \\
\hline SpeedXtract (SE) & $\begin{array}{c}140.7 \pm 50.5 \\
{[121.89-159.63]}\end{array}$ & $\begin{array}{c}0.77 \pm 0.22 \\
{[0.68-0.85]}\end{array}$ & N/A & $\begin{array}{c}63.33 \% \\
{[43.86 \%-80.07 \%]} \\
(19 / 11)\end{array}$ & $\begin{array}{c}100.00 \% \\
{[88.43 \%-100.00 \%]} \\
(0 / 30)\end{array}$ & $\begin{array}{l}2 \operatorname{hrs} 20 \\
\min ^{\mathrm{c}}\end{array}$ & $40 \min ^{\mathrm{c}}$ & $15.5^{\mathrm{e}}$ & $\begin{array}{l}6.5 \\
\mathrm{c}, \mathrm{e}\end{array}$ \\
\hline
\end{tabular}

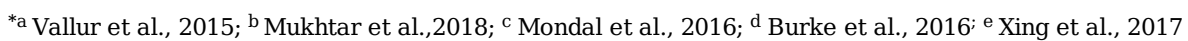

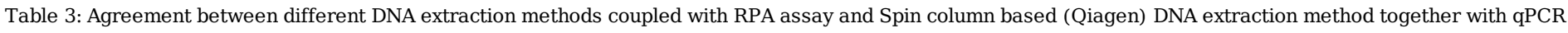
assay

\begin{tabular}{|c|c|c|c|}
\hline & Kappa (k) & Agreement & Mcnemar (p value) \\
\hline Q-RPA vs B\&S-RPA & 0.831 & Excellent & 0.06 \\
\hline Q-RPA vs SE-RPA & 0.692 & Good & 0.004 \\
\hline B\&S-RPA vs SE-RPA & 0.635 & Good & 0.34 \\
\hline Q-RPA vs Q-qPCR & 0.933 & Excellent & 0.50 \\
\hline B\&S-RPA vs Q-qPCR & 0.828 & Excellent & 0.38 \\
\hline SE-RPA vs Q-qPCR & 0.755 & Good & 0.02 \\
\hline
\end{tabular}

\section{Figures}




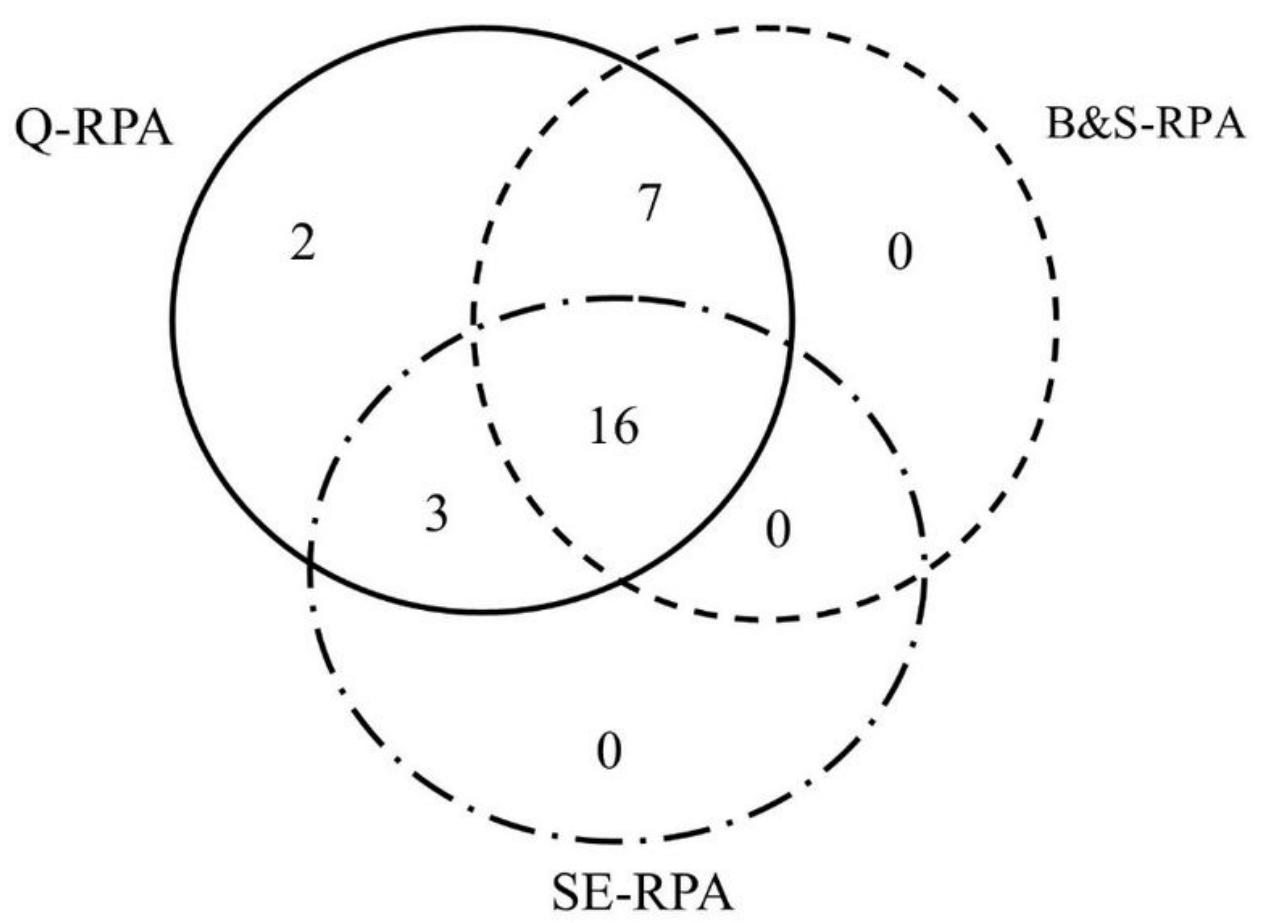

Two samples were negative by all three methods

Figure 1

Venn diagram depicting the distribution of Post kala azar leishmaniasis detected through Q-RPA, B\&S-RPA and SE-RPA. Among 30 skin biopsy samples from PKDL patients, total 28 were positive for L. donovani by Q-RPA, whereas, 23 and 19 samples were positive by B\&S-RPA and SE-RPA respectively.
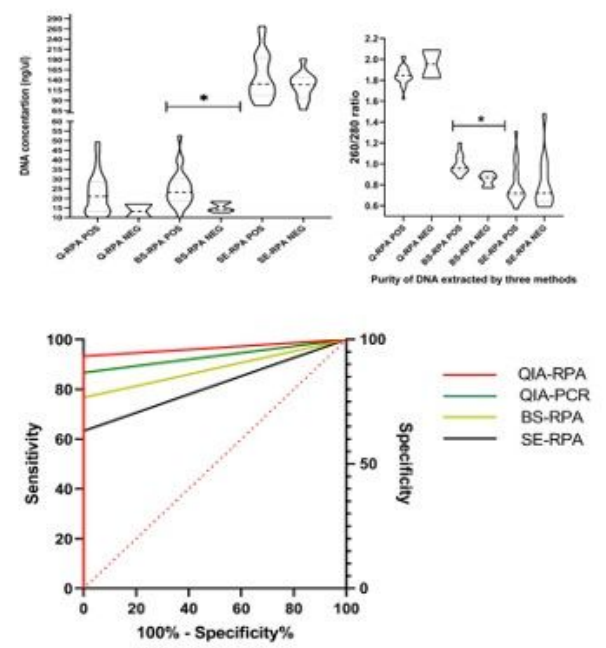

Figure 2

Illustration of the performance of three DNA extraction methods in coupled with RPA assay. In (A), the graph presented the concentration of DNA by the Spin column (Qiagen), Boil \& Spin and SpeedXtract extraction method, ${ }^{\star \star} p<0.001$. In (B), the purity of DNA through the Spin column (Qiagen), Boil \& Spin and SpeedXtract extraction method, ${ }^{* *} \mathrm{p}<0.0001$. In (C), Receiver operating characteristic (ROC) curve showing the diagnostic accuracy of the qPCR and RPA 
assay coupled with the Spin column based (Qiagen), Boil \& Spin and SpeedXtract extraction method for the detection of Leishmania parasites in skin biopsy samples. 